\title{
Changes of joint position sense in responses to upper trapezius muscle fatigue in subclinical myofascial pain syndrome participants versus healthy control
}

\author{
Mehdikhani Roya \\ Olyaei Gholam Reza \\ Shadmehr Azadeh \\ Hadian Mohammad Reza \\ Talebian Moghadam Saeed
}

Physical Therapy Department, School of Rehabilitation, Tehran University of Medical Sciences and Health Services: Piche Shemiran, Tehran, Iran

Corresponding author:

Olyaei Gholam Reza

Physical Therapy Department

School of Rehabilitation,

Tehran University of Medical Sciences and Health Services: Piche Shemiran

Enghelab Street, PO Box 1148965141

Tehran, Iran

Tel.: 0098-21-77528469

Fax: 0098-21-7534133

E-mail: Olyaeigh@sina.tums.ac.ir

\section{Summary}

Introduction: Fatigue of the neck muscles can lead to change in upper limb proprioception, motor patterns and kinematics. Restricted range of motion is a characteristic of myofascial trigger point. There is no evidence indicating an association of fatigue with restricted range of motion and non specific myofascial pain syndrome without underlying disease. This study evaluates cervical motions in flexion/extension, side bending and rotation before and after fatigue. Clinically, proprioception is evaluated using the Joint Position Sense Error (JPSE), which reflects a person's ability to accurately return his head to a predefined target after a cervical movement. This study design is randomized control trial.

Methods: 23 healthy right handed volunteers (11 males and 12 females) from a physical therapy clinic in rehabilitation faculty participated in the study.

Fatigue protocol was done, the recordings of pain threshold and intensity and kinematics, before and after fatigue, were all repeated.

Results: Neck muscle fatigue had different effects on neck kinematics for each group. JPSE in neck side bending repositioning was significantly greater following the upper trapezius muscle fatiguing protocol in a frontal plane.

Conclusion: The increased accuracy was most pronounced for movements directed towards the activated side. Hence, prolonged unilateral neck muscle contraction may increase the sensitivity of cervical proprioceptors. Cervical range of motion in side bending was the only variable associated with changes in neck pain.

Level of evidence: IIlb.

KEY WORDS: upper trapezius muscle, trigger point, fatigue, joint position sense error.

\section{Introduction}

Myofascial trigger points (MTrPs) are hyperirritable points within a taut band of skeletal muscle, which can be one of the primary reasons for pain and defect in the upper limb. Simons ${ }^{1}$ describes MTrPs as a tender point within a taut band of a skeletal muscle that is awful that is beginning with compression, contraction, or stretch and usually responds with a referred pain pattern away from the point. The greater identified MTrPs in the body are placed in the upper trapezius muscle.

Numerous sensory receptors required for proprioception are present in the neck muscles. Joint position sense (JPS) is the main method used to assess proprioception. JPS is regarded as the awareness of the position of various body parts for both passive and active movements in an open or closed chain environment $^{2}$.

JPS is considered as a major basis of proprioception. It is a person's ability to recreate and identify previous predetermined positions or ranges of motion (ROM) of a joint.

The error people make whilst reproducing the predefined position is defined as the joint position sense error (JPSE). The main measure to useful cervical proprioception is the $\mathrm{JPSE}^{3}$.

Malmstrom et al. (2009) showed that exact head-ontrunk orientation can be achieved without vestibular data. This means that proprioceptive information of the cervical spine is essential for head-on-trunk orientation. The cervical JPSE is assessed by measuring the ability of a blindfolded volunteer to accurately re- 
locate their head to the trunk relative to a predefined point (often the neutral position of the head) after a cervical movement.

Recent studies show that cervical ROM is impaired in disorders associated with MTrP. There is no evidence that indicating an association of fatigue about restricted ROM and nonspecific myofascial pain syndrome without underlying disease. This study evaluates where cervical ROM in flexion/extension, side bending and rotation before and after fatigue.

It has been shown that fatigue of the neck musculature can lead to change in upper limb proprioception, motor patterns and kinematics ${ }^{4}$. This may be for the reason that the high density of sensory receptors in the neck muscles that have neural networks to the vestibular and oculomotor systems, and show a main role in postural regulation ${ }^{5}$. The afferent feedback from muscles is affected by muscle fatigue while the cervical extensor muscle (CEM) fatigue alters the sensory feedback from the neck muscles to the central nervous system (CNS), thereby altering the position of the upper limb in respect to the neck ${ }^{6}$.

The response of mechanically sensitive non-spindle group II and III muscle afferents to muscle stretch and surface pressure is sensitized by their spontaneous discharge. This could result in increased muscle contraction, following submaximal muscle fatigue. Hayward et al. showed that free nerve endings which were sensitized to contraction remained so for 20-30 min after a fatiguing muscle stimulation. This fact implies that afferents might play an important role in the inhibition of motor neuron output following fatigue ${ }^{7}$.

Based on the anesthetized cat, it is possible to record the responses of afferents in groups III and IV to the sustained muscle contraction. A graded response was shown by one type of afferent to muscle contraction followed by the sudden onset as well as stretch (contraction-sensitive mechanical) and were composed mainly of group III rather than group IV afferents. Even after the period of muscle contraction, the other type of afferent had a delayed onset and continued to fire (unknown mechanism) ${ }^{8}$.

Based on the effects of prolonged muscle contraction and fatigue on afferent muscle feedback, there is a high tendency that fatigue will result in altered afferent feedback to the sensorimotor integration areas. It has been shown by earlier studies that altered input from the neck can affect cervical proprioception, and it is important to understand how fatigue, which occurs in a myriad of workplace and recreational settings, could impact head and neck proprioception.

Given the known effects of fatigue on the firing of muscle afferents, neck muscle fatigue would lead to changes in the afferent input from the neck for several minutes, after stopping the fatiguing task. If neck position sense can be altered by neck pain and altered postures, the possibility is very high that the altered afferent input resulting from neck muscle fatigue would also cause head and neck JPS. The objective of this study was to determine if a submaximal fatigue protocol targeting the upper trapezius muscle could alter the ability to recreate a previously presented cervical angle. This investigation involved an isometric submaximal fatigue protocol at $80 \%$ MVC until failure. It was assumed that the change in body schema, due to changes in the neck afferent feedback would result to an altered perception of the neck relative to the trunk and hence, increase the absolute error of the neck JPS and the error changes in the JPS is different in the upper trapezius muscle of healthy patients and the existence of myofascial trigger point in this muscle.

There is no study about the effect of fatigue on JPSE in subclinical myofascial pain syndrome participants. The aim of this randomized controlled trial was to prove that surface electromyography is able to detect upper trapezius latent myofascial trigger points through fatigue indices (median frequency and root mean square).

\section{Materials and methods}

\section{Ethical standards and study type}

This randomized control trial study reports baseline data from current prospective trials which were approved by the Tehran University of Medical Science Ethical Committee.

Each subject signed written informed consent prior to participant in the experiment.

\section{Participants}

Twenty-three healthy right handed volunteers (eleven males and twelve females) aged 18 years and older from a physical therapy clinic in rehabilitation faculty participated in the study. These patients initially presenting with chronic non specific neck pain were screened for MTrP.

Demographic characteristics of the patients are presented in Table I. Diagnostic criteria for detection of MTrP encompassed: (1) presence of a painful nodule in a taut band; (2) patient's pain recognition; (3) referred pain pattern and jump sign. These signs were selected as they, according to Lucas et al. ${ }^{9}$, display the highest reliability. Besides manual palpation, pressure pain threshold (PPT), is defined as the minimal amount of pressure that replaces the sense of pressure in to pain measured by a hand held algometry. It should be noted that the PPT for inclusion should been with values of $<3 \mathrm{~kg} / \mathrm{cm}^{2}$. VAS or visual analogue scale also has determined with values of $<30$. The presence of latent MTrPs was determined by an examiner with more than 7 years of experience in the diagnosis of MTrPs. Exclusion criteria encompassed radicular symptoms, acute traumata, and severe neurological, cardiovascular, psychological or inflammatory-rheumatic diseases.

In the control group, 23 healthy age-and-sex matched individuals (mean age and standard deviation, 29.12) were included. 
Table I. Demographic and clinical characteristics of patients $(n=46)$.

\begin{tabular}{llll}
\hline & Patients & Control group & $p$-value \\
\hline Age & 28.74 & 29.12 & $>0.05$ \\
Sex & 12 females, 11 males & 12 females, 11 males & $>0.05$ \\
BMI & 21.90 & 22.13 & $>0.05$ \\
\hline
\end{tabular}

\section{Instrumentation}

Angular variables were estimated by a digital camera (Sony Crop. Model number. DSC W390, made in Japan) placed 1 meter away from the subject at $\mathrm{C} 7$ level with a direct view of the subject's back in the frontal plane. The camera collected range of motion and kinematics data at the rate of 25 frames per second. The markers used to measure the segment angles were attached to the subjects as follows: three circular markers were attached to the $\mathrm{C} 7$, right and left acromion process.

\section{Protocol}

The subjects sited on a chair, their wrists hanged in the side of their body. The upper trapezius, levator scapula, and sternocleidomastoid (SCM) muscles were examined bilaterally for MTrPs. To define a MTrP, the examiner palpated the muscles in search of a taut band in the muscle, within which they looked for a nodule. If a nodule was located in angular region of upper trapezius muscle in dominate side, the examiner pressed the nodule for few seconds and asked the subject whether it evoked pain. The examiner instructed the subject as following: I am going to apply pressure, tell me when you feel a minimal amount of pain, then applied pressure to the MTrP with the algometer, at a rate of approximately 1 $\mathrm{kg} / \mathrm{cm}^{2} /$ second. ${ }^{10}$ After introducing the task to the subjects and making sure of the accuracy of the maneuver, subjects MTrP performed three $10 \mathrm{sec}$ trials separated by $20 \mathrm{sec}$ between them. The maximum numbers of maximum voluntary contraction (MVC) were limited to three. Mean of the achieved numbers selected as a MVC.

The goal of the fatigue procedure used in this study was to fatigue the muscles involved in the shoulder elevation, especially the prime mover of the shoulder elevation, i.e., the upper trapezius.

Before doing the test PPT and pain intensity and alignment of head and neck was assessed. Photography of the patient neck position in neutral and maximum opposite side bending was done for three trials. Prior to commencing the current study, a pilot study was performed using three healthy participants. The fatigue protocol consisted of holding an isometric upper trapezius muscle contraction with $50 \%$ of MVC, and it took an average of $16 \mathrm{~min}$ for participants to fatigue. It was difficult for participants to maintain their focus and hard to motivate participants over this lengthy time period. Thus, an $80 \%$ MVC protocol was selected, which resulted in an average of $3 \mathrm{~min}$ to fatigue.

The force level corresponding to $80 \%$ of the MVC was chosen because the protocol aimed to trigger muscle fatigue.

To confirm the presence of muscle fatigue, the fatigue confirmation task was performed using the dynamometer and EMG. First, the peak torque (PT) of the shoulder elevation during maximal voluntary isometric contraction (MVIC) was measured at the target angle with the subject in the sitting position with the shoulder positioned at $90^{\circ}$ of abduction and the elbow positioned at $90^{\circ}$ of flexion Subsequently, the EMG data during the performance of maximum PT before the fatigue task was recorded for $3 \mathrm{sec}$ while visually confirming the torque output. At the same time as we visually confirmed that the subjects output the target torque, we started recording the EMG. Each measurement was performed in duplicate.

The head was kept in the same position as the trunk and the vertebral column. During the test, the subjects were asked to look forward with no cervical and trunk rotation, extension or flexion. The subjects were asked to sustain a unilateral $80 \%$ MVC isometric shoulder elevation until the force gauge monitor showed $50 \%$ of MVC at least for 3 minutes. After a light warm-up, subjects performed three (MVC) tests in the position described above.

Feedback of the force level was provided. The perceived exertion was rated by the subject at the start and after each minute of the contraction. Force and EMG signals were recorded during the MVC and sustained contractions.

Although no subjective assessment of fatigue was made, the subjects showed exhaustion at the end of the protocol. The highest value was used to define the workload, i.e., $80 \%$ of the MVC force. The fatigue protocol used in this study was an intermittent isometric contraction exercise (10 sec of workload; $20 \mathrm{sec}$ rest). The force requirement was displayed on a computer monitor positioned at eye level and in front of the participant to ensure that a neutral head position was maintained throughout the protocol.

Following fatigue protocol, the recordings of pain threshold and intensity and kinematics were all repeated. At the completion of the protocol, they performed a MVC test intending to calculate the loss of force and the median frequency from the power spectrum of the upper trapezius EMG signal. The test was 
performed one time pre and post fatiguing, respectively. Prior to the pre-test the participants received standardized oral instructions by one of the examiners and visual instructions in a video. In case of poor initial performance these instructions were repeated up to three times and the test was demonstrated by one examiner. The participants performed the posttest immediately after completing an isometric endurance test to fatigue the upper trapezius muscle. Figure 1 shows the three trials for achieve ROM and kinematic data.

JPS was measured as the participant's ability to recreate a previously presented angle at the cervical. After the two pre-fatigue sets, participants performed the fatigue protocol. Immediately following the fatigue protocol, participants performed one set of three post-fatigue cervical JPS trials with three separate target angles. We chose three trials per set because the possibility existed that participant might start to recover from the fatigue protocol if additional trials were performed. Three trials still allowed for the calculation of the average error, while minimizing learning effects and ensuring that any changes resulting from the neck muscle fatigue were likely still present.

\section{Absolute, constant, and variable error}

Based on the procedure ${ }^{6}$, the accuracy of the angle of reproduction was determined using three parameters for each condition. In order to determine the direction and magnitude of the position error, the following parameters were calculated:

1. absolute error was defined as the overall deviation between a presented angle and target angle without considering the direction of the error;

2. variable error was defined as a measure of the consistency between trials and is the SD of the mean constant error;

3. constant error was defined as the deviation between the presented and reproduced angle, considering the direction of the error.

In each set, the average of the three trials was used for error calculation.

\section{Statistical analysis}

Repeated measures analysis of variance (ANOVA) with pre-planned contrasts to the first baseline was performed for each variable and used to measure JPS between pre- and post-fatigue conditions (SPSS v19, IBM Corporation, Armonk, New York, USA). Statistical significance was set at $p \leq 0.05$. Microsoft Office Excel 2010 (Microsoft Corporation 2010; Redmond, Washington, USA) was used to calculate absolute, variable, and constant neck joint position sense error. In addition, two sample $t$ tests for data with unequal variances were calculated to evaluate force level and time to fatigue.

\section{Results}

All the participants were able to complete the experiment, with no issues or interaction with the experimenter. No significant differences between gender and intervention parameters were found in any of the two groups. Neck muscle fatigue had different effects on neck kinematics for each group. JPSE in neck side bending repositioning was significantly greater following the upper trapezius muscle fatiguing protocol in a frontal plane (i.e. side bending) $(p<0.001)$. However, there were no significant differences in the same variable in sagittal (flexion/extension) and transverse plane (rotation-left and rotation-right) movements.

\section{Participants}

Average demographics of the interventional and control groups are summarized in Table I. There were no other statistically different demographics between the groups.

\section{Reliability of the three repeated measures for each trial}

The reliability of the three trials for neck side bending was very high, with interclass correlations ranging from 0.82 to 0.99 . Table 2 shows demographics of interventional and control groups and table 3, 4 and 5

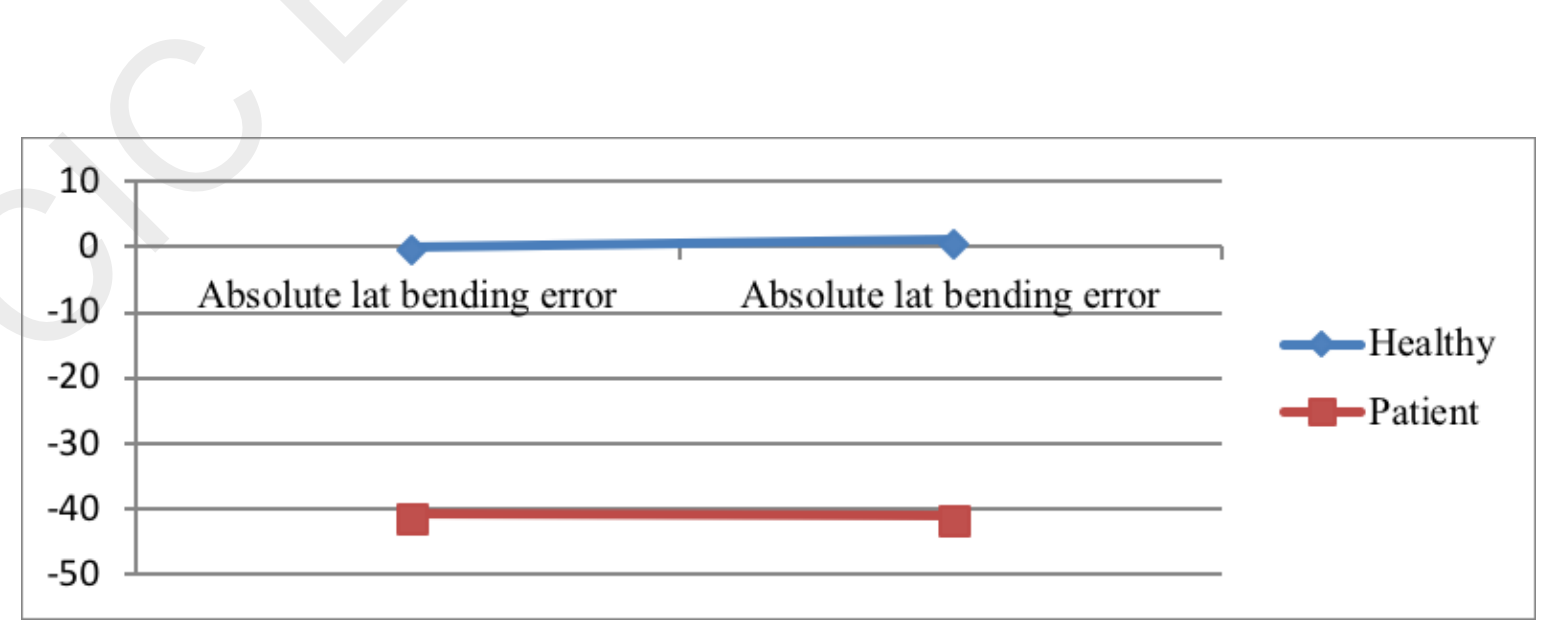

Figure 1. Effect of fatigue on absolute lateral bending error according to group. 
Table II. Demographics of the interventional and control groups.

\begin{tabular}{lll}
\hline & Patient group & Control group \\
\hline Age & 0.73 & 0.88 \\
Weight & 0.82 & 0.99 \\
Height & 0.97 & 0.76 \\
BMI & 0.72 & 0.87 \\
Mean Lat Bending & 0.51 & 0.39 \\
Mean Flex & 0.12 & 0.91 \\
Mean Rot & 0.74 & 0.55 \\
Mean K Lat Bending & 0.60 & 0.75 \\
Mean K Flexion & 0.34 & 0.32 \\
Mean K Rot & 0.52 & 0.134 \\
\hline
\end{tabular}

BMI, body mass index; Lat, lateral; Flex, flexion; Rot, rotation; K, kinesthesia.

Table III. Repeated measurement. Absolute error lateral bending before and after fatigue.

\begin{tabular}{lllll}
\hline Factor & Mean Square & F & Sig & $\begin{array}{l}\text { Partial Eta } \\
\text { Squared }\end{array}$ \\
\hline Factor 1 (absolute lat bending before \& after) & 38274.332 & 1066.946 & .000 & 0.961 \\
Factor $1^{*}$ Group & 12.444 & 0.347 & 0.559 & 0.008 \\
Factor $1^{*}$ Gender & 100.268 & 2.795 & 0.102 & 0.061 \\
\hline
\end{tabular}

Table IV. Repeated measurement. Absolute error lateral bending before and after fatigue.

\begin{tabular}{lllll}
\hline Factor & Mean Square & F & Sig & $\begin{array}{l}\text { Partial Eta } \\
\text { Squared }\end{array}$ \\
\hline Factor 1 (relative lat bending before \& after) & 35207.856 & 912.624 & .000 & 0.955 \\
Factor $1^{*}$ Group & 3.511 & 0.091 & 0.764 & 0.002 \\
Factor $1 *$ Gender & 149.432 & 3.873 & 0.056 & 0.083 \\
\hline
\end{tabular}

show errors before and after fatigue. Variable error was significantly differing between groups but absolute and relative errors were similar. Figures 2, 3 and 4 show the graph of these errors. Figure 5 and 6 show effect of fatigue on lateral bending errors. There were no significant JPE differences between the neck pain and control groups for any of the flexion/extension and rotation motions.

\section{Discussion}

This study compared cervical JPS error accuracy and the consistency between the upper trapezius muscle trigger point subjects and the control group. As such, forty-six subjects were asked to perform the fatigue test. Absolute, constant and variable errors were used to evaluate proprioception accuracy and consistency. 
Upper trapezius muscle fatigue in subclinical myofascial pain syndrome participants

Table V. Repeated measurements. Variable error lateral bending before and after fatigue.

\begin{tabular}{lllll}
\hline Factor & Mean Square & F & Sig & $\begin{array}{l}\text { Partial Eta } \\
\text { Squared }\end{array}$ \\
\hline Factor 1 (absolute lat bending before \& after) & 11.644 & 1.138 & 0.292 & 0.026 \\
Factor $1^{\star}$ Group & 93.150 & 9.103 & 0.004 & 0.175 \\
Factor $1^{\star}$ Gender & 26.783 & 2.617 & 0.113 & 0.057 \\
\hline
\end{tabular}

The variable error which is indicative of the consistency of results for each subject was higher in the myofascial pain syndrome group.

Neck muscles, in particular the small suboccipital deep muscles have a very high density of muscles spindles and are, therefore, essential contributors to neck proprioception ${ }^{11}$.

Optimal movement precision requires afferent input from muscle spindles ${ }^{12}$.

Without excluding other contributing factors, it has been suggested that disturbed proprioceptive input, as a result of altered input from the muscle spindles, could constitute an important pathophysiological mechanism in chronic musculoskeletal pain conditions of various origin ${ }^{13}$.

It is known that muscle spindle afferents convey position and movement information in ensembles of afferents and that the information content in such ensembles is reduced when the fusimotor control of the muscle spindles is disturbed by high activity in group III and IV muscle afferents ${ }^{14}$.

By using an animal model, it was found that the excitation of chemosensitive nociceptors in cervical facet joints and muscles induce reflex activation of fusimotor neurons which alter the static and dynamic sensitivity of the muscle spindles ${ }^{15}$.

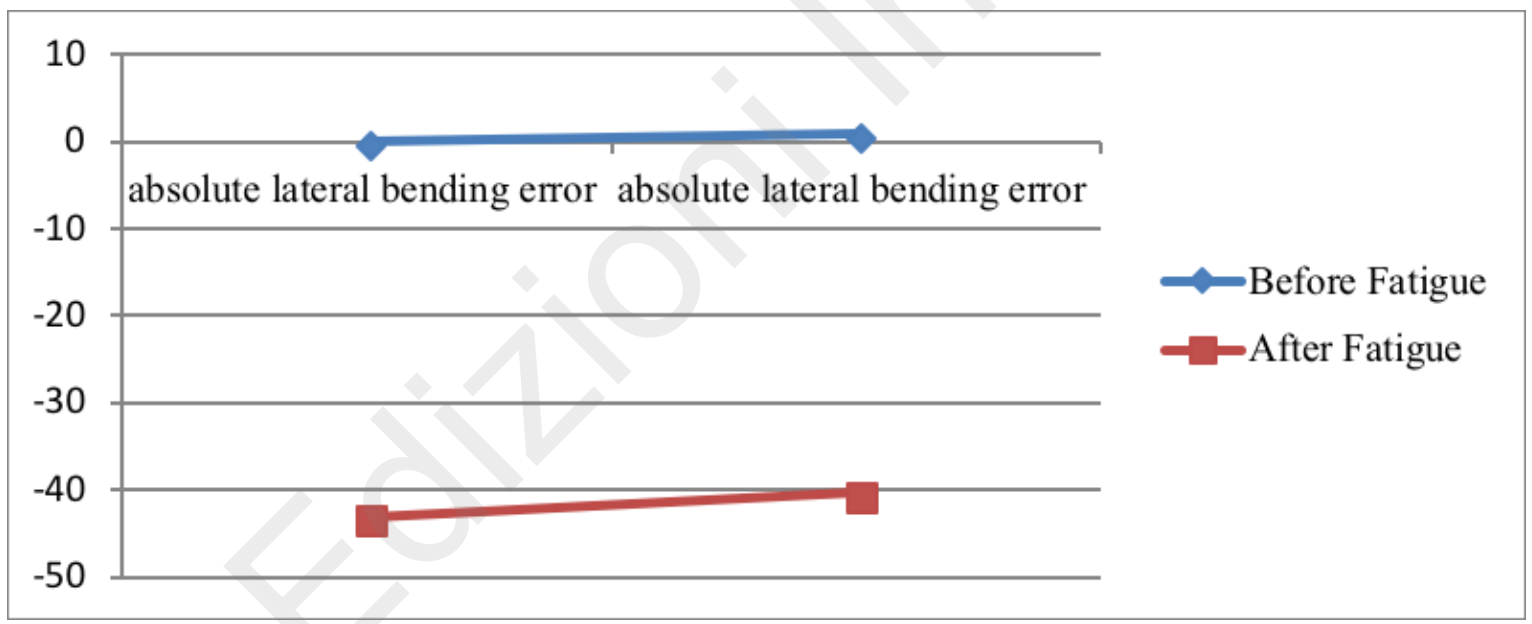

Figure 2. Effect of fatigue on absolute lateral bending error according to gender.

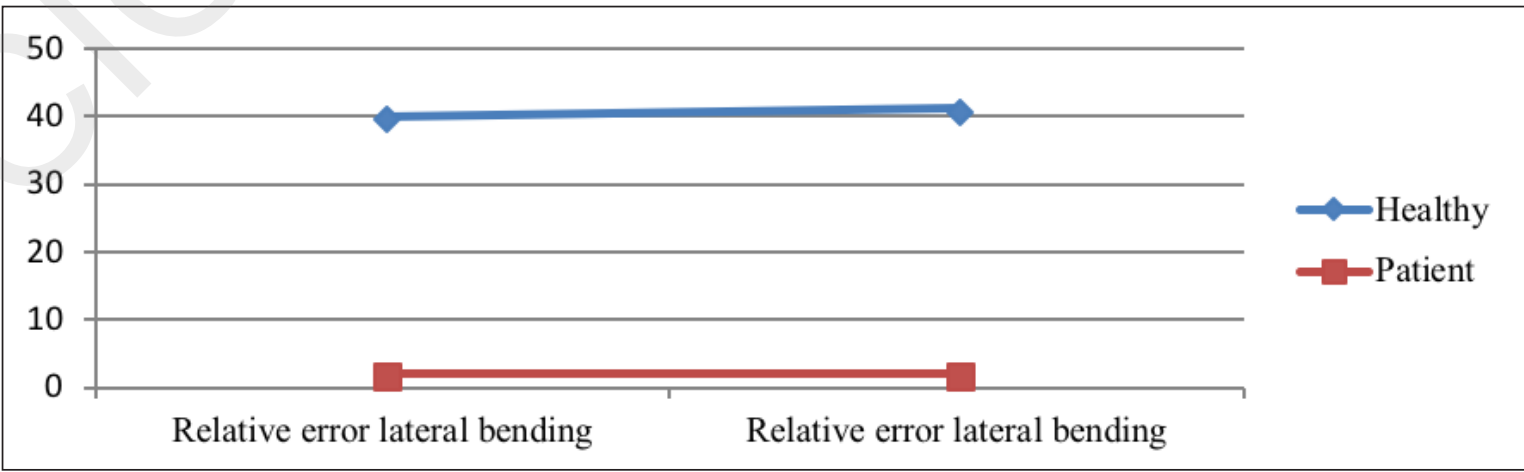

Figure 3. Effect of fatigue on relative error of lateral bending error according to group. 


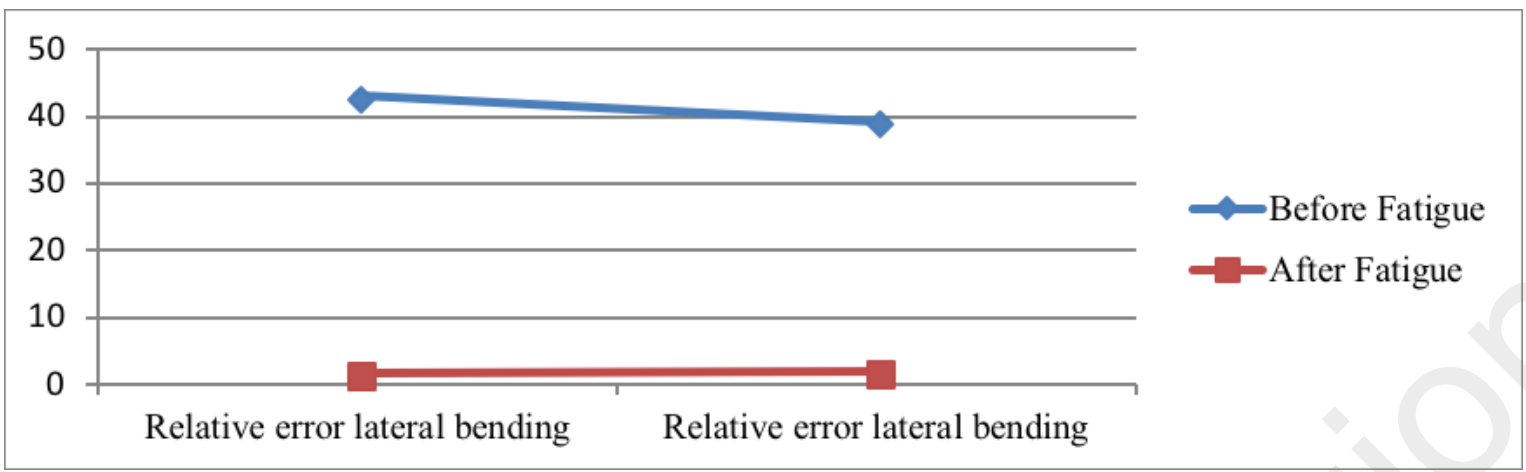

Figure 4. Effect of fatigue on relative error on lateral bending error according to gender.

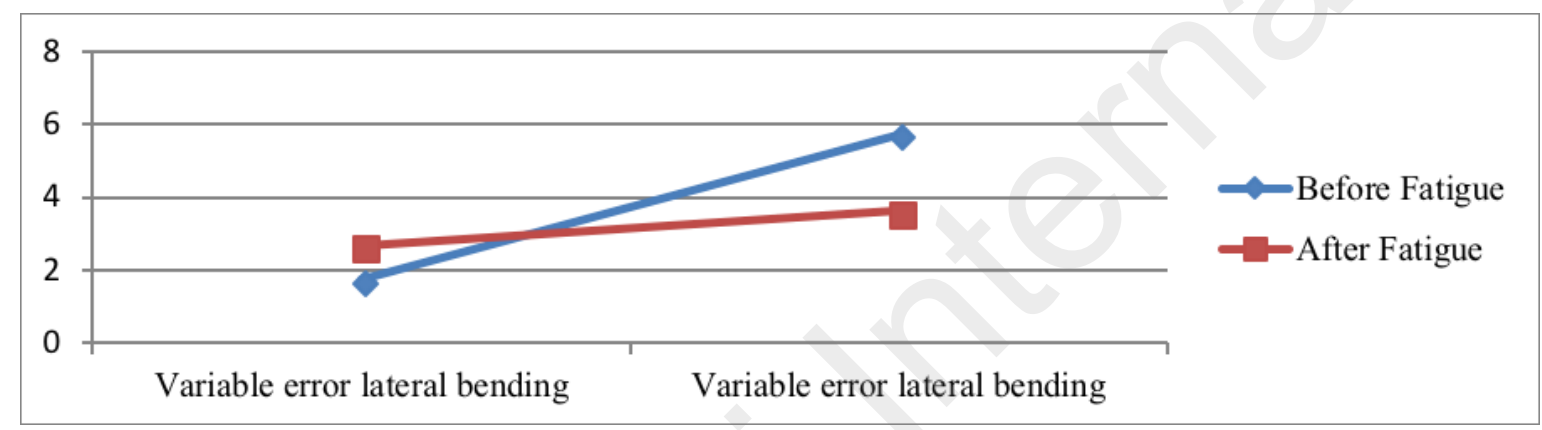

Figure 5. Effect of fatigue on variable lateral bending error according to group.

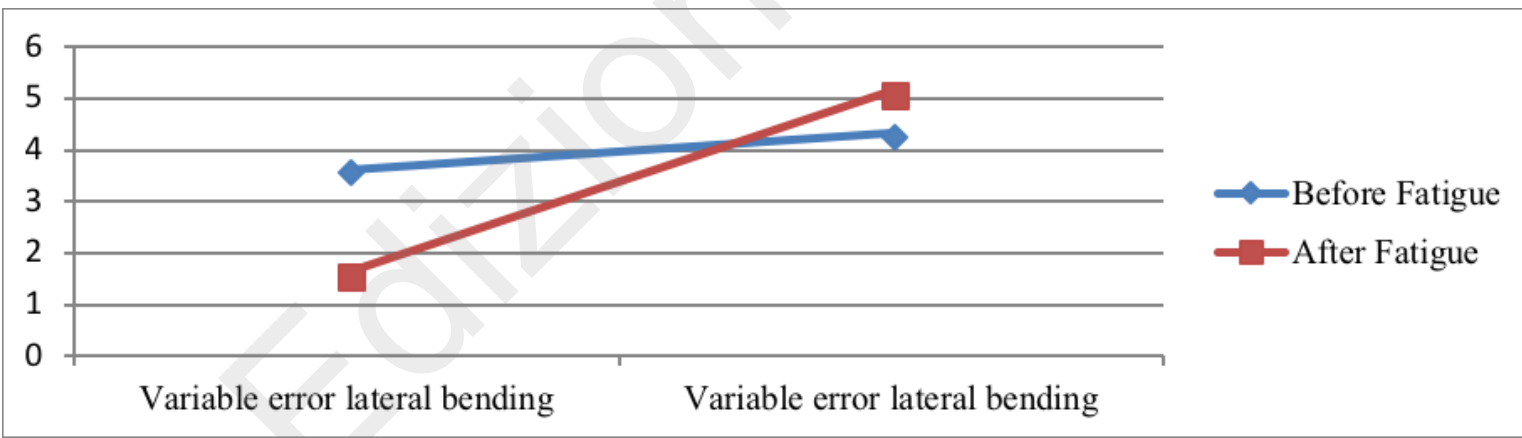

Figure 6 . Effect of fatigue on variable lateral bending error according to gender.

Moreover, common to most of these studies is that the average absolute error of a set of trials was used as the outcome variable. Absolute error is also regarded as a non-linear combination of constant (CE) and variable error (VE). In a repositioning task, CE denotes the mean deviation from the target; it also represents the point of subjective equality, while VE stands for the variability of the matching trials around the $\mathrm{CE}^{16}$.

There is proof that $\mathrm{CE}$ reflects the intrinsic systematic biases in the motor control system, and that VE is the result of the acuity of sensorimotor processes, i.e. the position sense acuity. It is noteworthy that according to psychophysical literature, the latter is in line with the general definition of sensory discrimination threshold in adjustment tasks. The Jerk index and ROM-Variability denote novel parameters in attempts to quantify motor control disturbances in patients with chronic neck pain. The findings that both parameters were significantly larger among patients than among asymptomatic subjects strengthen previous results indicating movement irregularities in chronic neck pain ${ }^{17}$.

Due to the fact that large ROM-Variability, in similarity with large repositioning error, reflects the variability of the end position of the rotation, it appears that the disturbed position sense acuity is involved in both increased VE and ROM-Variability.

The mechanisms responsible for increased jerk index are still unclear.

However, it is believed that unconscious motor con- 
trol mechanisms rather than psychological processes might be responsible. Increased extent of co-activation of neck muscles could generate jerkier movements, as could biomechanical disturbances due to skeletal or soft tissue injuries. Finally, jerky and slow movements might be a reflection of motor control strategies dominated by feedback control, perhaps as a consequence of reduced acuity of the proprioceptive input, and continuous feedback corrections, or as a result of disturbed feed forward control mechanisms. The observation of larger repositioning errors in patients with chronic neck pain than among asymptomatic subjects is in agreement with previous findings ${ }^{18}$.

In the present study, the significantly larger VE indicated reduced repositioning acuity. Among the patients, there was no systematic over- or underestimation (i.e., CE) of the matched target position.

These findings suggest that the increased repositioning errors observed in triggered point neck pain are a result of poor position sense acuity as a result of the disturbed proprioceptive input, rather than of a systematic bias in the motor control systems at central levels. This interpretation is based on fundamental psychophysical data showing that the variability (i.e., $\mathrm{VE}$ ) of the matching errors in an adjustment task reflects the threshold for sensory discrimination ${ }^{16}$.

Thus, if the signal-to-noise ratio of the information transmitted in the muscle spindle afferents is reduced in response to increased noise in the fusimotor signal, it would be reflected in decreased proprioceptive acuity. Such disturbances of the fusimotor control could be triggered by for instance, a massive activation of mechanosensitive nociceptors that may result from the soft tissue injuries occurring in a whiplash trauma, and by activation of chemosensitive joint and muscle nociceptors which are likely to occur during inflammation or when the blood flow in the muscle is reduced $^{19}$.

Accordingly, the diminished mechanoreceptor and muscle spindle function, and the central misinterpretation of the proprioceptive inputs could lead to the loss of cervicocephalic kinesthetic acuity.

Regarding repositioning error, these study findings are in line with findings in adults with neck pain. Two systematic reviews of studies comparing neck proprioception between adults with neck pain and asymptomatic controls concluded that adults with neck pain are worse than asymptomatic controls at head-toneutral repositioning tests ${ }^{19}$, suggesting impaired joint positioning sense, one of the components of proprioception. Repositioning errors for rotation-to-neutral varied between 3.3 and $6.1^{19}$.

Several mechanisms may contribute to the altered neck position sense, including: the effects of pain itself on both nociceptors and mechanoreceptor activity locally, at the spinal cord and within the central nervous system; disturbed sensitivity of the cervical joint and muscle receptors, or by inflammatory mediators 20 .

In addition, central sensitization (as suggested by the
PPTs and other findings of the present study discussed in the previous paragraph), is believed to be associated with reorganization of the somatosensory cortex in chronic pain syndromes, including changes in the cortical representation of painful body sites ${ }^{21,22}$. This study explored the position accuracy during frontal head reposition movements as well as the corresponding EMG responses of the upper trapezius muscle fatigue in subjects with and without neck pain. Earlier studies showed the position accuracy of the sagittal head-to-neutral tests in young healthy subjects (18-30 years old) and in middle-aged subjects with neck pain (40-65 years old) 22 .

The following mechanisms explain the reasons for the poor position sense in subjects with neck pain. The perception of egocentric space in adults includes kinesthetic information from visual input and vestibular afferents, as well as abundant muscle spindles and mechanoreceptors of the facet joint capsules in the neck region. With normal visual and vestibular afferents, the peripheral proprioceptive inputs are relayed to the central nervous system for regulating joint movements through the activation of muscles ${ }^{23}$.

A modified interpretation of neck proprioceptive signals in the central nervous system could also result in an offset in the egocentric reference frame and interfere with the central control over the activation of muscles ${ }^{24}$.

No comparative study was found to report the VE in subjects with the upper trapezius muscle trigger point.

Compared with those studies, the healthy subjects in this study showed a similar range of $A E$ and $C E$, while subjects with the upper trapezius muscle trigger point group showed much higher VE. The smaller reposition errors in this study could be attributed to the fact that only young adult subjects were recruited. One possible reason for a higher amount of VE in triggered pointed subjects is that myofascial pain syndrome may lead to the use of different motor synergy strategies when trying to relocate the head position and this can lead to a higher variability in responses and therefore a higher VE error. In other words, due to changes in muscle length and orientation followed by a change in joint position, as a result of poor habitual posture, the outcome of bad variables overcomes good variables, when performing a particular task more than once 25 .

\section{Conclusions}

It seems that different types of perturbations from the normal condition such as disease, age, or abnormal posture, can result in variable responses in different trials. However, there was no significant difference between both groups in absolute and constant errors, indicating that fatigue has no effect on joint position sense accuracy.

An interesting finding was that experimental group had slightly lower, but insignificant $A E$, compared to 
control group. Our results suggest that variable error is a more accurate and delicate indicator to be used for studying joint position sense. Future studies are recommended to investigate the role of other postural deficiencies such as scoliosis, or lordosis, on joint position sense.

One of the limitations of our study was that only a limited number of shoulder joint muscles were evaluated. The effects of other muscles attached to scapula or related to shoulder elevation and any deficits in these muscles on movement quality were not assessed. As mentioned before, the upper trapezius muscle was chosen, because of its key role in dynamic stability of scapular during upper limb elevation and the strong possibility for the presence of LMTrPs in UT muscle.

Another limitation of this study was that the amplitude of the muscle activations during movement and muscles related to shoulder elevation were not taken into account.

Moreover, all participants in this study were females with a very narrow age range and the findings cannot be extrapolated to all female age groups.

\section{Acknowledgements}

This is a part of PhD thesis. The Authors are grateful to Mr Mohammad. Taghi Moghadam Fard, Chairman of the Board of Iran Exhaust Co, who built the setup of study and carried out part of the experiment.

We appreciate the Postgraduate Studies and Research Program, Tehran University of Medical Sciences, for providing the generous grant to carry out this research. We also acknowledge the generous assistance of the staff and students of the Rehabilitation school, Tehran University of Medical Sciences.

\section{Compliance with ethical standards}

\section{Conflict of interest}

The Authors declare that they have no conflict of interest. The results of the study are presented clearly, honestly, and without fabrication, facilitation, or inappropriate data manipulation.

\section{Ethics}

The Authors declare that this research was conducted following basic ethical aspects and international standards as required by the journal and recently update in ${ }^{26}$.

\section{Ethical clearance statement}

Ethical clearance was obtained from The Tehran University of Medical Science Ethics Committee and was adhered to throughout the study.
Ethics Committee number IR.TUMS.VCR.REC 1395.803; 2016/10/22 and all subjects signed an informed consent form prior to their inclusion. IRCT2017011426346N2

\section{References}

1. Simons DG. Clinical and etiological update of myofascial pain from trigger points. Journal of musculoskeletal pain. 1996;4(12):93-122.

2. Riemann BL, SM Lephart. The sensorimotor system, part I: the physiologic basis of functional joint stability. Journal of athletic training. 2002;37(1):71

3. Armstrong B, et al. Head and neck position sense. Sports medicine. 2008;38(2):101-117.

4. Zabihhosseinian $M$, et al.Neck muscle fatigue alters upper limb proprioception. Experimental brain research. 2005;233 (5):1663-1675.

5. Jull G, et al. Retraining cervical joint position sense: the effect of two exercise regimes. Journal of Orthopaedic Research. 2007;25(3):404-412.

6. Knox JJ, PW Hodges. Changes in head and neck position affect elbow joint position sense. Experimental brain research. 2005;165(1):107-113.

7. Hayward L, et al. Effects of muscle fatigue on mechanically sensitive afferents of slow conduction velocity in the cat triceps surae. Journal of Neurophysiology. 1991;65(2):360-370.

8. Mense S, M Stahnke. Responses in muscle afferent fibres of slow conduction velocity to contractions and ischaemia in the cat. The Journal of Physiology. 1983;342(1):383-397.

9. Lucas $\mathrm{KR}$, et al. How common are latent myofascial trigger points in the scapular positioning muscles? Journal of musculoskeletal pain. 2008;16(4):279-286.

10. Reeves $\mathrm{JL}$, et al. Reliability of the pressure algometer as a measure of myofascial trigger point sensitivity. Pain. 1986;24(3):313-321.

11. Kulkarni V, et al. Quantitative study of muscle spindles in suboccipital muscles of human foetuses. Neurology India. 2001;49(4):355.

12. Bergenheim $\mathrm{M}$, et al. Proprioceptive population coding of twodimensional limb movements in humans: I. Muscle spindle feedback during spatially oriented movements. Experimental brain research. 2000;134(3):301-310.

13. Johansson $\mathrm{H}$. Epilogue: An integrated model for chronic workrelated myalgia 'Brussels Model'. Chronic work-related myalgia: Neuromuscular mechanisms behind work-related chronic muscle pain syndromes. 2003.

14. Ribot-Ciscar E, et al. Proprioceptive population coding of limb position in humans. Experimental brain research. 2003;149 (4):512-519.

15. Thunberg $\mathrm{J}$, et al. Influences on the fusimotor-muscle spindle system from chemosensitive nerve endings in cervical facet joints in the cat: possible implications for whiplash induced disorders. Pain. 2001;91(1-2):15-22.

16. Gescheider G. Psychophysics: the fundamentals Lawrence Erlbaum, Mahwah, NJ. 1997.

17. Clark FJ, et al. A metric for assessing acuity in positioning joints and limbs. Experimental brain research. 1995;107 (1):73-79.

18. Revel M, et al. Cervicocephalic kinesthetic sensibility in patients with cervical pain. Archives of physical medicine and rehabilitation. 1991;72(5):288-291.

19. Sjölander $P$, et al. Spinal and supraspinal effects of activity in ligament afferents. Journal of Electromyography and Kinesiology. 2002;12(3):167-176.

20. Le Pera $\mathrm{D}$, et al. Inhibition of motor system excitability at corti- 
cal and spinal level by tonic muscle pain. Clinical Neurophysiology. 2001;112(9):1633-1641.

21. Tsay A, et al. Sensing the body in chronic pain: a review of psychophysical studies implicating altered body representation. Neuroscience \& Biobehavioral Reviews. 2015;52:221-232.

22. Lee $\mathrm{H}-\mathrm{Y}$, et al. Test-retest reliability of cervicocephalic kinesthetic sensibility in three cardinal planes. Manual therapy. 2006;11(1):61-68.

23. Fasold $\mathrm{O}$, et al. Proprioceptive head posture-related processing in human polysensory cortical areas. Neuroimage. 2008; 40(3):1232-1242.
24. Paulus I, Brumagne S. Altered interpretation of neck proprioceptive signals in persons with subclinical recurrent neck pain. Journal of rehabilitation medicine. 2008;40(6):426432.

25. Latash ML, JG Anson. Synergies in health and disease: relations to adaptive changes in motor coordination. Physical therapy. 2006;86(8):1151-1160.

26. Padulo J, Oliva F, Frizziero A, Maffulli N. Muscles, Ligaments and Tendons Journal - Basic principles and recommendations in clinical and field science research: 2016 update. MLTJ. 2016;6(1):1-5. 